\title{
Evaluating the utility of the FamCHAT ethnocultural nursing assessment tool at a Canadian tertiary care hospital: A pilot study with recommendations for hospital management
}

\author{
Gina MA Higginbottom ${ }^{1}$, Magdalena S. Richter ${ }^{1}$, Susan Young ${ }^{2}$, Lucenia M. Ortiz ${ }^{2}$, Stella D. \\ Callender ${ }^{2}$, J oan I. Forgeron ${ }^{2}$, Michele L. Boyce ${ }^{2}$ \\ 1. Faculty of Nursing, University of Alberta, Alberta, Canada. 2. Alberta Health Services, Kingsway Ave., Edmonton, \\ Alberta, Canada.
}

Correspondence: Gina MA Higginbottom. Address: 6-10K University Terrace, University of Alberta, Edmonton, Alberta, Canada, T6G 2T4. Telephone: 780-492-6761. Fax: 780-492-1926. E-mail: gina.higginbottom@ualberta.ca

Received: November 8, 2011

DOI : $10.5430 /$ jnep.v2n2p24
Accepted: January 16, $2012 \quad$ Published: May 1, 2012

URL: http://dx.doi.org/10.5430/jnep.v2n2p24

\section{Abstract}

Objectives: The multicultural nature of Canadian society and the decline in health of immigrants after relocation to Canada prioritizes a commitment to ensure equity in health care access and outcomes. An important feature shaping health care access and outcomes is the reception of culturally safe and competent care. Partnering with senior management at a tertiary care hospital, this pilot study aimed to investigate whether an identified cultural assessment tool, the Family Cultural Heritage Assessment Tool (FamCHAT), validated for use in a rural primary care setting, was suitable for use in tertiary care. The objectives were to investigate: 1 ) whether using the FamCHAT enabled the nurses to elicit assessment information from their patients who represent diverse ethnocultural groups, and 2) the nurses' perspectives on the practical use of this form in their clinical practice.

Methods: Nurses purposively selected from the Women's, Surgery and Medicine units were asked to complete the FamCHAT form with all patients admitted during a three-month period in 2009. Focus group interviews were then held to learn the nurses' perspectives related to the form's constructs and its use in their practice. The data from the completed FamCHAT forms were tabulated and analyzed using descriptive statistics to determine the extent of completion and accuracy, and the sample characteristics. The interview data was analyzed using qualitative analytical software (ATLAS.ti Scientific Software Development, GmbH, Germany) and Roper and Shapira’s framework for analysis of ethnographic data.

Results: The nurses filled out forty-four FamCHAT forms with patients having a diverse ethnocultural profile. In many forms, several questions were either left blank or answered incorrectly with regard to the guidance notes containing categorical answers for the variables of family size and language. Nine nurses participated in two focus groups $(\mathrm{n}=4$ and $\mathrm{n}=5$ ) and one in an individual interview. Five themes emerged from the qualitative data analysis: feasibility of using the FamCHAT in acute care practice; ethnocultural awareness needs of the participating nurses; perspectives of nurses about patient concerns; potential for enhancement to nursing care assessments; and suggestions for enhancing and facilitating a new tool or approach. Nurses participating in the interviews thought that the constructs within FamCHAT could be useful 
for enhancing nursing assessments in their practice, but thought the tool was too long, was repetitive to some of their current assessment data, and had questions many patients were uncomfortable answering. One option suggested in both focus groups was to embed the most useful constructs into existing assessment frameworks. Some of the variables were thought to be of limited relevancy for some nursing specialties.

Conclusions: The findings provided valuable information for the hospital management in their efforts to revise nursing assessment tools. Consideration is being given to integrating some of the constructs into their existing nursing assessment, with recognition that each unit might benefit from different approaches. Other, systematic, approaches to enhancing cultural competency also need to be considered. This study highlights the importance of validating practice tools for use in settings that differ from those used for their original development.

\section{Key words}

Nursing assessment, FamCHAT, Cultural assessment tool, Cultural competency

\section{I ntroduction}

\subsection{Health equity priority for Canada's multicultural society}

Historically, successive Canadian governments have actively encouraged immigration and naturalization in attempts to impact strategic demographic, economic, social, humanitarian and security goals ${ }^{[1,2]}$. The 1971 Multiculturalism Policy of Canada and the Multiculturalism Act of 1985, both communicating equality of law and opportunity as well as respect regardless of race, ethnic origin, language or religious affiliation, have further contributed to a shift in migration trends ${ }^{[3]}$. Moreover, the ethnocultural profile of Canada's population has changed considerably over recent years, partly due to persistently low-birth and high immigration rates ${ }^{[4]}$. Indeed, the 2006 census found that $19.8 \%$ of the total population was foreign born ${ }^{[5]}$.

While Canadians are among the healthiest in the world, significant disparities are present and several reports have addressed this issue in recent years ${ }^{[6-8]}$. In a longitudinal study, $15-20 \%$ of newcomers reported difficulties in accessing health care after arriving in Canada ${ }^{[9]}$, and asylum seekers/refugee immigrants may be at a particular disadvantage when negotiating the health care system ${ }^{[10]}$. Moreover, evidence largely from health survey data but also empirical research supports a healthy immigrant effect, whereby the health status of immigrants (although typically not refugees) upon arrival is high yet converges downwards over time towards that of the native-born population ${ }^{[11,12]}$. While substantial diversity exists within and between immigrant populations, commonality may exist in terms of challenges encountered during access to and navigation and receipt of health services, and in experiences of migration, ethnocultural adaptation and acculturation. The multicultural nature of Canadian society together with the healthy immigrant effect prioritize a commitment to ensure equity in access and outcomes within its health care systems, partly by through ensuring practitioners are enabled to practice culturally competent care.

\subsection{Need for culturally competent care}

Where culturally appropriate care is not delivered, studies demonstrate a negative trajectory of events ranging from simple miscommunication to life-threatening incidents ${ }^{[13,14]}$. Some patients may hold ethnocultural beliefs and values that could interfere with their adherence to recommendations from medical practitioners ${ }^{[15]}$. Additionally, poorly handled cross-cultural issues were thought by many physicians to result "often" in other consequences including longer office visits, delays obtaining informed consent, ordering of unnecessary tests, and lower quality of care ${ }^{[16]}$. The efficient and effective provision of health care services for all ethnocultural groups can thus be argued to have important patient safety, health economic, and risk management dimensions. Additionally and perhaps most apparent is that cultural competence improves patient satisfaction, since many ethnocultural groups value relational quality thus highly interpersonal care. 
Facilitating health care providers to provide culturally competent care for immigrant populations is critical for the health and wellbeing of Canada's richly diverse population. Indeed, registered nurses in Canada are professionally committed to provide culturally competent care, implied within their code of ethics' value of fairness which describes care "in response to clients' needs and (which is) respectful of 'race' ethnicity, culture, spiritual beliefs, marital status, gender, sexual orientation, age, health status, lifestyle or physical attributes" ${ }^{[17]}$. One useful definition of cultural competence is "the ability to understand and work effectively with patients whose beliefs, values, and histories differ from one's own" [18] Expanding this Giger et al. ${ }^{[19]}$ offer the definition of "having the knowledge, understanding, and skills about a diverse cultural group that allows the health care provider to provide acceptable cultural care. Competence is an ongoing process that involves accepting and respecting differences and not letting one's personal beliefs have an undue influence on those whose worldview is different from one's own. Cultural competence includes having cultural general as well as cultural specific information so the health care provider knows what questions to ask". Although cultural competence in its entirety encompasses the qualities of compassion, empathy, and responsiveness to the needs, values, and expressed preferences of individual patients ${ }^{[20]}$ and should include systemic policies (largely structural and related to linguistic services), steps to encourage cultural competency may include provision of interactive resources such as assessment tools which provide cultural specific knowledge to health professionals and may facilitate understandings. Moreover, a cultural competency perspective is perhaps most relevant for those institutions where individuals from diverse ethnocultural backgrounds seek care.

\subsection{Background}

Senior staff at a tertiary care teaching hospital located in one of the city's most diverse neighbourhoods became aware of the need for current nursing assessments to take in account the ethnocultural dimensions of nursing care provision, in order to optimize interactions during diagnosis, treatment, and management of a health event. The management felt that nursing care should thoughtfully and comprehensively include orientation to the ethnocultural background and related needs of their patients and their families. The orientation was anticipated to occur during the initial phase of assessment. It is for these reasons that a research team was formed late in 2007 to identify an ethnocultural assessment tool with potential suitability for implementation, and to perform a pilot study using the identified tool in practice. The team consisted of two academic researchers, the hospital's director of nursing, one unit manager and two clinical nurse educators of the hospital, and the health authority's multicultural coordinator.

\subsection{Review of availability and empirical basis of ethnocultural models and assessment tools for nursing practice}

Although various disciplines have contributed theoretical and conceptual frameworks ${ }^{[21]}$ which may assist nurses and health care practitioners in understanding the social positioning of their diverse ethnocultural patient groups, many of the frameworks have not tended to underpin specific care assessment or delivery tools used in clinical nursing practice. In nursing, models of cultural competency have tended to be seminally informed by the transcultural nursing theory of Leininger ${ }^{[22,23]}$. The research team performed a critical review of the literature to identify an empirical perspective on the use of a model or tool within clinical nursing practice specifically devised for use with diverse ethnocultural groups, and which might thus be transferable to the care context at this particular hospital ${ }^{[22]}$. Using a systematic approach with a comprehensive search strategy, many of the abstract citations described the development/validation of tools such as questionnaires for use with specific disease states or procedures, and not related to cultural aspects of care, and for evaluating various training programs in the topic. Very limited literature explicitly described models or tools (including assessment tools) incorporating cultural assessment for use in nursing practice. After full article review, eight papers were selected that described eight models/tools for use in ethnocultural nursing practice (see Figure 1). Many of these assessment tools and models are based on clinical experience but have never been tested in any manner for use within nursing practice ${ }^{[22]}$. One of the tools, an open-ended questionnaire entitled the Family Cultural Heritage Assessment Tool (FamCHAT), was developed to add cultural dimensions to an existing validated family health assessment tool (FAMTOOL) and evaluated for reliability with 131 Kansas clients ages 8-80 in a rural family practice ${ }^{[24,25]}$. The tool was 
derived from themes regarding cultural diversity arising from longitudinal studies with a culturally diverse rural population of immigrants and their descendants between 1895 and 1995. Face and content validity have reportedly been completed with lay representatives and an expert panel, respectively ${ }^{[25]}$. The research team agreed that, while only empirically tested for use in a primary care setting, the FamCHAT tool was succinct and user-friendly in nature, with underpinning principles highly pertinent and transferable to the acute/hospital setting.

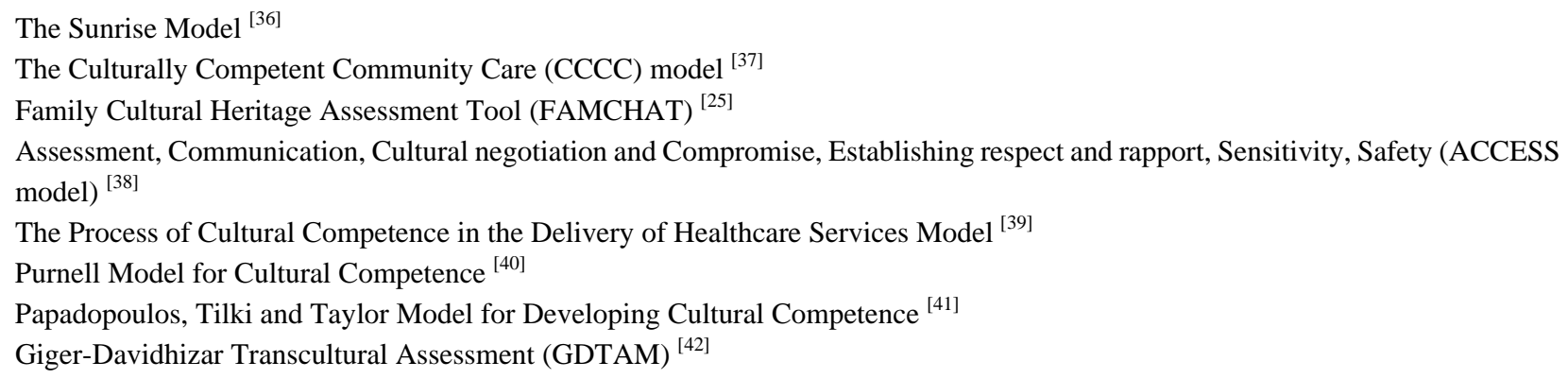

Figure 1. Names of identified models/tools in cultural competency having some empirical basis for nursing practice.

\subsection{Aim}

This paper describes a pilot study to investigate whether an identified cultural assessment tool, the Family Cultural Heritage Assessment Tool (FamCHAT) and validated for use in the primary care setting, was suitable for use in the acute care hospital setting to potentially help enhance ethnocultural dimensions of nursing assessment and optimize care. The objectives were to investigate: 1) whether using the FamCHAT enabled the acute care nurses to elicit assessment information from their patients, who represent diverse ethnocultural groups, and 2) the nurses' perspectives on the practical use of this form in their clinical practice.

\section{Methods}

A pilot study was designed having two parts consisting of : i) completion of the FamCHAT form by nurses of three hospital units (Women's, Surgery, and Medicine) with all admitted patients over a three-month period, and ii) subsequent focus group interviews with participating nurses. Institutional ethical approval was granted by the ethics board overseeing research at this hospital and all participating staff and patients provided informed consent to participate in this study. The potential exists for participants (employees) who are recruited by employers to experience either actual or perceived coercion, thus the research team was sensitive to this and ensured that the participants (nursing staff and patients) were aware that their participation was of a voluntary nature and their anonymity would be assured during presentations and publications.

\subsection{Research setting}

In 2006, the population of the relevant city reached one million with 189,775 people identifying themselves as foreign born, 113,825 immigrating before 1991, and newcomer groups from Asia (particularly Southeast Asia) and the Middle East being the largest ${ }^{[26]}$. Those in visible minority groups totalled 175,295 (17\%), with the top four groups being Chinese, South Asian, Black, and Filipino ${ }^{[26]}$. The hospital under consideration for this study is a tertiary care teaching hospital located in one of the city's most diverse neighbourhoods. The region is home to many immigrant newcomers and older European immigrants from Italy, Poland and the Ukraine; as well it is a hub for numerous businesses with owners of diverse ethnocultural origin. 


\subsection{Revision of FamCHAT form}

The research team thought that the format of the original form did not allow for enough space to provide full answers, so they included more white space in the form while maintaining the single-page format (see Figure 2). Furthermore, the category "income" was replaced with "occupation", and the phrase "family tree" was included to clarify the meaning of "genealogy”. Permission for these changes was granted from its developer.

FAMCHAT - A Family Cultural Heritage Assessment Tool

Please respond to the following questions in the space provided so that we might better serve you and your family

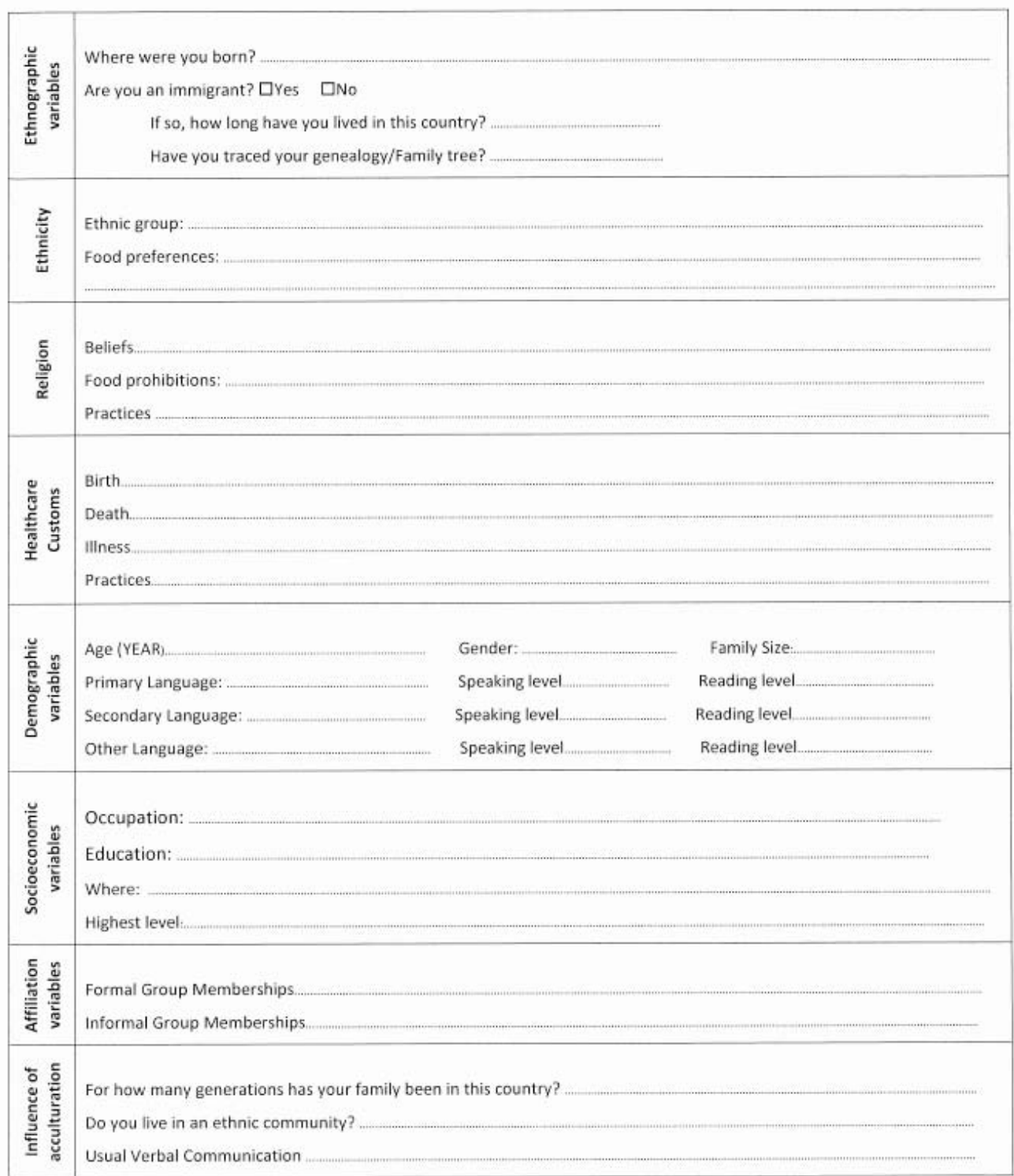

Figure 2. Revised FamCHAT form. The main revision was related to the format as the original form was thought to not have enough space for to provide full answers. Furthermore, the category "income” was replaced with "occupation”, and the phrase "family tree" was added to clarify the meaning of "genealogy". Permission for these changes was granted from its developer Dr. Janice Unruh Davidson of Chamberlain College of Nursing, USA. 


\subsection{Sampling, recruitment, and training}

Nurses were purposively sampled from the Women's, Surgery, and Medicine Units. The Women's unit sees a large number of immigrant women, and the Medicine unit had the highest request rate as logged in Admissions for in-house interpretation services (in 2006 which was the last year this interpretation service was provided). Surgical procedures require collection of complex information prior to, during, and after treatment, which can prove problematic when caring for patients who have a limited understanding of English.

Posters and information letters left within nursing units introduced the project to potential nurse participants. The study was also championed by the three clinical staff who were also research co-investigators. Sessions were provided to interested nurses to offer background to the research, orientation regarding cultural competency, and training related to their expected role in the study. Sessions were facilitated by two research team members in each unit during the day, evening and night shifts (during coffee breaks and supplying refreshments) to accommodate all nursing shifts. Each 30 min session engaged and obtained consent from the staff, and trained them with respect to ethical issues, document storage, and the administration of the FamCHAT. It was important to ensure that the nurses were familiar with the key concepts in the form and had some experiential learning of administering the form in a safe environment; experiential activities were facilitated in which participating nurses practiced using the form with each other during the training session. Guidance notes for use when filling in the FamCHAT form were provided and described. The notes contained general definitions of some of the terms (immigrant, ethnicity, ethnic community) and specific categorical answers which the team required for the questions related to family size (extended, nuclear, lives alone), speaking levels (fluent, partially fluent, non-fluent), and reading levels (fully comprehend, partially comprehend, unable to comprehend the written word). Since the participating nurses acted as designates for the investigators when gaining informed consent from the patients prior to the completion of the FamCHAT, the orientation included discussion of the principles of informed consent while stressing the voluntary and confidential manner of the research.

\subsection{Data collection}

The nurses were instructed to complete the FamCHAT form during their initial patient assessments. The original copy of the completed form was kept separate from the patient's records in a locked cabinet, while a carbon copy containing no identifying information (name, address, date of birth or health care/hospital codes) was forwarded to the research team. The answers to all questions were tabulated in Excel (Microsoft Office Excel 2007). Answers which did not match the categorical answers specified in the guidance notes for the variables of family size and spoken and written language were recorded as incorrectly answered.

Participating nurses were asked to provide feedback on the tool within focus group interviews (FGIs). The FGIs were conducted at lunch time in a room on the hospital site and lunch was supplied. The investigators or trained research assistants used a topic guide for the FGI, with questions related to the characteristics of the form (e.g. completeness as related to culturally specific information, problem areas, and perceived appropriateness for their patients), the usefulness and feasibility of implementing the form in their acute care practice, and how and if it should be changed or revised to be more context specific. All interviews were digitally recorded and transcribed verbatim by a professional transcriptionist.

\subsection{Data analysis}

The data collected in the FamCHAT forms was used to answer in part our first objective of whether the FamCHAT enabled nurses within their acute care practice to elicit culturally specific information from their patients. We considered all of the FamCHAT constructs to provide culturally specific information so the main priority was to determine the ability of the nurses to actually elicit suitable answers to the respective variables. The data from the FGIs was used to answer both objectives but largely the second which related to what the nurses' perspectives were on the use of the form in their clinical practice. 
The data from the submitted FamCHAT forms was tabulated, with quantitative data (age, gender, family size, language fluency and comprehension, and immigrant status) reported using descriptive statistics (Microsoft Office Excel 2007) and trends of open-ended answers described. No comparisons were made of data collected in the different units. Qualitative data from the FGIs were stored, managed, classified and ordered with the aid of ATLAS.ti qualitative data analysis software package (Atlas.ti Scientific Software Development GmbH, Berlin, Germany). Roper and Shapira's [27] framework for analysis of ethnographic data was utilized, which involves coding for descriptive labels, sorting for patterns, identification of outliers or negative cases, forming generalizations, constructs and theories, memoing, and reflective remarks. The process represented an iterative process, whereby preliminary interpretations were challenged and data are revisited in light of further data collection and new insights. The research team wrote field notes after each interview and during their work on the units (some team members being practicing nurses) and engaged in research team meetings to enable articulation of reflexive dimensions in the data analysis. Attention was paid to misrepresentation of data.

\section{Results}

The revised FamCHAT was piloted in June through August 2009. During this time period, 44 completed forms were returned to the research team by the three units (Medicine 54\%, Surgery 44\%, and Women's 2\%). Two FGIs (n=4 Surgery unit; $n=5$ Medicine unit) and one individual interview (Women's unit due to sole participant) were completed. Of the fifteen nurses who completed forms, twelve were born in Canada (most with Canadian or Northern European self-assigned ethnicity), two in the Philippines, and one in the UK.

Table 1. Data from demographic section and related to immigration

\begin{tabular}{|c|c|}
\hline Age (mean \pm SD, range) & $66 \pm 20,23-91$ \\
\hline Gender (M/F; \%) & $52 / 46$ \\
\hline Family size (\%) & \\
\hline - $\quad$ Extended & 6 \\
\hline - $\quad$ Nuclear & 0 \\
\hline - $\quad$ Lives alone & 0 \\
\hline - Incorrectly answered & 80 \\
\hline - $\quad$ Blank & 14 \\
\hline Primary Language (\%) & \\
\hline - $\quad$ English & 80 \\
\hline - $\quad$ Other* & 20 \\
\hline - $\quad$ Speaking level: & \\
\hline - $\quad$ Fluent & 57 \\
\hline - $\quad$ Partially fluent & 0 \\
\hline - $\quad$ Non-fluent & 0 \\
\hline - Incorrectly answered & 6 \\
\hline - $\quad$ Blank & 38 \\
\hline - $\quad$ Reading level: & \\
\hline - $\quad$ Fully comprehend & 23 \\
\hline - $\quad$ Partially comprehend & 0 \\
\hline - Unable to comprehend the written word & 2 \\
\hline - Incorrectly answered & 68 \\
\hline - $\quad$ Blank & 7 \\
\hline Secondary Language (\%) & \\
\hline - $\quad$ English/French & 27 \\
\hline - $\quad$ Other & 23 \\
\hline - Blank or "none" & 50 \\
\hline Immigrant $(\%)^{\dagger}$ & 25 \\
\hline - $\quad$ Length of residency (range; years) & $9-58$ \\
\hline
\end{tabular}

*Other primary languages included Dutch, Cantonese, Cree, Finish, French, Polish, Spanish, and Twi.

†Countries of birth included Holland, Netherlands, Hong Kong, El Salvador, Finland, Germany, Ghana, Japan, Poland, and Scotland. 


\subsection{Ability of nurses to elicit culturally specific information from the patients}

The descriptive statistics for the demographic variables (age, gender, family size, and speaking and reading levels) and immigrant status and length of residency are presented in Table 1. The open-ended answers to the other variables are described below and organized by the sections of the FamCHAT.

\subsubsection{Ethnographic \& ethnicity variables}

Fifteen participants (34\%) had, and 15 (34\%) had not traced their family tree; this question was left blank in 14 forms. Figure 2 shows the variety of ethnic groups represented, although two forms had the answers "not applicable", one indicated "none", and another five had the question unanswered. The purpose of the question on food preferences is to gain an understanding of ethnicity-specific food preferences, such as vegetarian or kosher foods which might influence culturally related satisfaction with hospital diet or educational interventions. Despite this, many of the answers reflected health concerns as indicated by "no salty food" or "low-fat diet".

\subsubsection{Religion}

Most of the answers (75\%) indicated Christian faith classified by the team as "Christian", "Catholic" (which included Roman Catholic, Ukrainian, Greek Catholic), or "Protestant” (including Anglican, Baptist, Lutheran, Orthodox Reformed, United Church). Three forms recorded "no faith" and three were blank. Most of the answers to the question of food prohibitions were "no", "none”, or "n/a". The question on practices was largely left blank or answered with "no" or "n/a", with the exception of one comment of "elders to be involved to promote ethical passing".

\subsubsection{Health care customs}

Similar to the construct of religion, many of the forms had no answers for these questions or answers of "not", "none", and "N/A". Some forms had brief notes related to birth customs surrounding circumcisions (against and favouring) and preference for baptism, to death customs related to (not) prolonging life by mechanical means and having a religious figure or elder present, and to preference for having family provide supportive or palliative care.

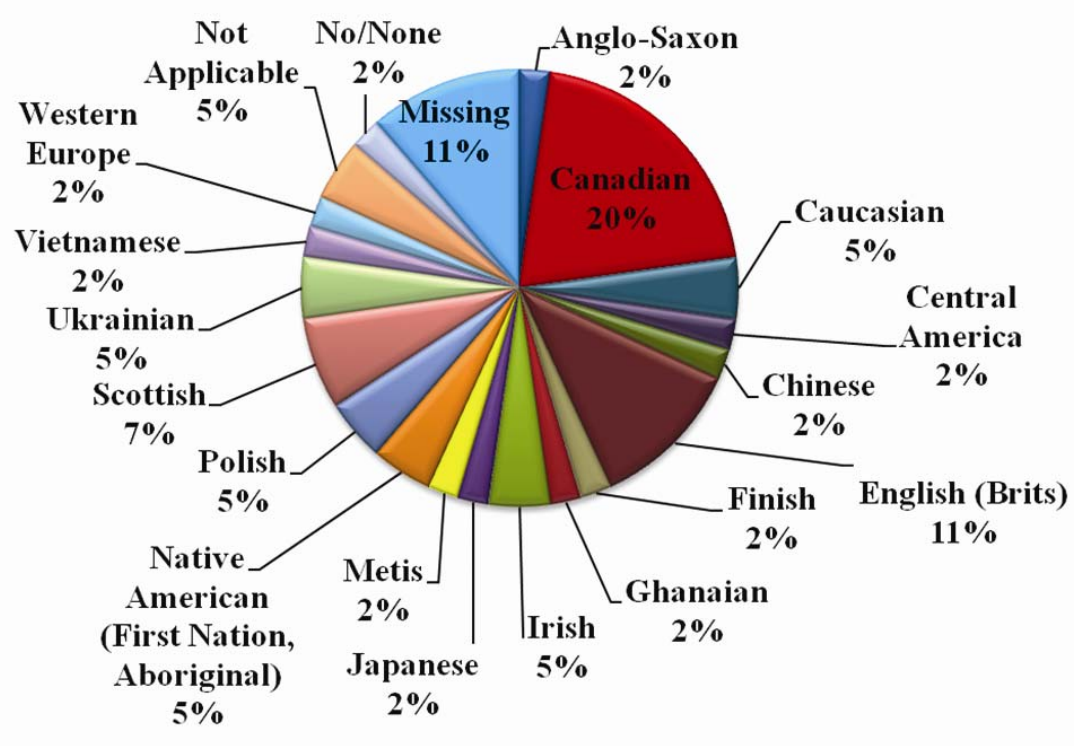

Figure 3. Self-assigned ethnicity of patients completing the form 


\subsubsection{Socioeconomic variables}

The occupations of the patients were varied as expected. Although the mean age of the patients was 66 years, only 23\% responded that they were retired. Twenty-two percent of patients had not achieved high school graduation. The number of patients who graduated high school in Canada (73\%) reflects the study population's high rate of Canadian-born patients. Thirty-two percent responded that they had some post-secondary education in trades school, college or university.

\subsubsection{Affiliation and influence of acculturation variables}

Answers related to these variables were highly varied and did not seem to reflect any specific ethnicity. Many of the completed answers related to formal group memberships related to churches $(n=6 / 31)$ or stated "none” $(n=21 / 31)$.

\subsection{Qualitative findings}

Five themes emerged from the analysis of the FGI and semi-structured individual interviews:

- Feasibility of using the FamCHAT in practice;

- Ethnocultural awareness needs of the participating nurses;

- Perspectives of nurses about patient concerns;

- Potential for enhancement to nursing care assessments;

- Suggestions for enhancing and facilitating a new tool or approach.

\subsubsection{Feasibility of using the FamCHAT in practice}

The general consensus in both FGIs was that the form was too long and complicated for use with their patients. Participants in one FGI mentioned that they felt the FamCHAT to be too time consuming to administer with their patients.

For me personally, it's okay... the only thing is, if we can like, lessen, too many questions, like because it takes so much time, like it's the time, you know ... we are so always like very, very busy and you ask them to do too many questions like this, it's kind of something frustrating. FGI Medicine unit

Two participants also commented that FamCHAT was complicated to administer, largely because some of the patients seemed not to have adequate levels of education and English literacy which were required.

...reading level, because some, a patient of mine before, he said he was in grade five, and I said 'Can you read', he said 'No, I can't read'. FGI Medicine unit

... but don't you think it's important that we know that? That a people can't, a person can't read? Especially um if it comes to the use of medicine, you send them home with instructions. I'm just wondering how you feel about that... FGI Medicine unit

Additional consensus related to a concern of using the tools during the night shift, when priority was on "settling" the patients. Moreover, the health of some patients greatly limited their ability to convey the information:

...the patients that I have admitted have had severe dementia, so (I: Right) it was even, we couldn't even get just a history, their medical history, so... FGI Surgery unit

\subsubsection{Ethnocultural awareness needs of the participating nurses}

Nursing is a profession that requires building mutual trust with patients. This is particularly important since hospital in-patients are disconnected from their extended family and friends with whom they usually live. In various settings the 
care of the patient would be enhanced by nurses having a better understanding about customs and traditions of different ethnocultural groups. This issue was discussed in length by the participant who completed the individual interview.

I think it's important to understand culture, in nursing care, having worked in labour and delivery and seeing many cultures deliver over the years, it does help to understand and anticipate, their reactions that they're anticipating, their pain, uh who's making decisions, who should be present in the room, what should happen if a birth or, even a stillborn. Different things over the years that I've observed and it does help to understand culture. I think it would be valuable. Individual interview

Matters related to food, religion, birth and death of different cultures were assessed as top priority for enhancing awareness.

I think it's really good to be able to know their food preferences, their religious beliefs... regarding birth and death, and that's quite important cause that happens here a lot. I imagine a headache most of the time. So that would be what I would say, some of this stuff is really, it's nice to know. Individual interview

One main area of ignorance was that regarding religious beliefs which dictate needs for specific care provision. One FGI participant of the Medicine unit had experienced a situation related to gender issues within the Orthodox-Jewish culture.

Well, we one time had patient on this unit who was, can't remember, the Orthodox-Jewish I think, and they had this belief that women during their menstrual period should not be giving care by a man. Now, we would've never known this. FGI Medicine unit

While most of the nurses confirmed their appreciation of the large diversity of patients admitted to this hospital, a few commented on the inability at times to learn and remember specific beliefs, values, and customs from particular groups of patients. The challenge at times seemed too great:

We have different types of ethnicity on the unit where, we're not too sure what their values, and beliefs and their customs are ...because there's so many different,... ethnic groups out there for us to be able to try and remember, what to do with them all, it's really difficult. Individual interview

An additional and major hurdle was the communication abilities of many patients. It was surprising that there are still some immigrants who do not speak English despite immigrating to Canada many years ago. Much of time there was a need to rely on the patients' families to relay information.

\subsubsection{Perspectives of nurses about patient concerns}

In the views of most of the nurses (and through correspondence with a research team member who was a nurse manager), a significant challenge when attempting to fill in the form was that some patients (particularly immigrants) seemed unwilling to provide their personal information due to apparent or stated suspicion regarding the motives of collecting such data. They seemed to be of the view that this tool did not protect their privacy. Therefore, in some but not all cases it was perceived that the individual may not be prepared to reveal their personal information to other people due to lack of trust and pre-migration history. Moreover, the patient may be under the assumption that this type of revelation might lead them to be sent back to their own country.

They don't want to tell us when they get here, because they don't want to be sent back, they have fear and a scare, so they're not so happy sometimes to give you, all the information, for a lot of different reasons. They, you know they have a right to be distrustful, I guess. Because of what they've come out of, and maybe what they've had to deal with, where they are, and they don't understand our medical system. FGI Surgery unit 
Apparently, different techniques ("tactics") were used in attempt to reassure patients that the purpose of the tool was for enhancing understanding rather than any for categorization/labelling in any manner.

Other participants described one of the drawbacks of the form as its apparent duplication with other routine questions asked during admission. It was stated that the patients indicated frustration with having to answer the same questions twice:

\section{Like [paraphrasing a patient] "I answered a whole bunch of questions the other day". FGI Surgery unit}

I just found though, usually when they come to the unit, they're, they've just been bombarded with questions downstairs, they don't feel like answering this all over again. So if it could be stretched out into a couple days, you know, they're more ... open to answer... well some would just clam right up, roll over and go to sleep, 'Look I've been an, answered all this downstairs, why do I need to do it again?... Well now it's, that has kind of changed, but I find even some are, they are so confused... FGI Medicine unit

The last portion of this quote takes the issue further by noting that since patients are not in their usual environment and have compromised health status, having too many questions to answer may result in confusion. The following quote further illustrates this:

Uh, only did one and he was able to do it so, most our patients here are confused, too sick no family member coming in I think that's why we don't get to do much of it. Cause I, I know like they were saying that we not, like you know the amounts of patients we have doesn't coincide on how many we do, and they're too sick, they're confused, no family member so we can't really do any follow-up on them. FGI Medicine unit

Another concern brought forth by two participants regarding patient acceptance of the form was that that patients (mainly newer immigrants) seemed uncomfortable when asked questions on some subjects such as educational achievement. It was thought that some immigrants may think that having better educational qualifications than required for their current employment status (due to foreign credentialing issues) is a source of embarrassment.

\subsubsection{Potential for enhancement to nursing care assessments}

One participant indicated that she was very excited when she first came to know of this form, and thought that its use would be very important since the hospital serves a highly diverse multicultural population. Utilization of the FamCHAT form may be regarded as a necessity for accessing their patients' health needs:

I believe it's a very necessary tool in our environment. We have such a multicultural population in our facility and I was excited when I saw this tool. Individual interview

It was portrayed that although many nurses have a high level of nursing knowledge and expertise, it was doubtful that the majority regularly facilitate culturally sensitive health care provision despite working within an organization where it can be critically important to understand in depth the needs of patients.

Several FGI participants indicated how the tool could potentially enhance their care. One nurse from the surgery unit commented that:

...it would give us a greater understanding for what a patient expects from us as well. The expectations... Because sometimes we come with a preconceived idea about how this course will go, and until someone has the opportunity to tell us that you know, this is what their expectation was, we have no idea. FGI Surgery unit 
The nurses from the surgery unit agreed that the questions around health care customs were "a big star". One nurse also made reference to the potentially greater suitability of some of the constructs in certain specialties, for instance asking about traditional norms around birth may be of little relevance for the majority of surgical patients. Alternatively, the nurses said that they might need to "filter" some variables with certain patients, such as those around death beliefs for the young patient with a fractured ankle as compared to the elderly lady with a fractured hip. Another nurse reflected that:

I think there's a lot of stuff on this I would, if they were to be a newly admitted I would ask right away, but some of these other things, I would maybe ask them throughout their stay. FGI Surgery unit

\subsection{Suggestions for enhancing and facilitating a new tool or approach}

Many issues brought forth during the interviews related to length, time consumption and literacy requirement of the FamCHAT. Several participants thought that consideration should be given to refining and shortening the tool, incorporating either checklists or individual, precise and short questions. The FGI with the participants of the Medicine unit had the most to comment on this topic.

A member of the Medicine unit firmly suggested that it is better to omit the occupation and education sections in the FamCHAT form. She further noted that it is better to remove questions associated with socioeconomic index:

I personally would omit the occupation section, 'education where highest level' part, if I had my pick, that would be something I would omit. What about education? ...I probably wouldn't ask that either. ...I wouldn't ...I wouldn't either...Even the level...because you can kind of figure from their job...Even the level of education, it would know the level of education....P: I think that whole thing should be scrapped, socio-economics, probably changed. FGI Medicine unit

This participant suggested that when the tool is amended, it would be better to refine the questions reflecting ethnocultural norms related to religion and ethnicity, and to change the order of the questions:

Potentially mind you, can combine these, but potentially the, in the area of the religion, ethnicity, that also may, may require more. It depends on the culture; some of them have a lot more customs and things, than others. FGI Medicine unit

It is, other than like I said, I'd probably ask the questions in a different order. You know like, we talked about rearrange the set up. FGI Medicine unit

Other members of the Medicine's unit FGI also made suggestions surrounding religious beliefs, death, food habits, and more importantly issues related to cultural restriction of blood transfusion.

Because like you, you know as we said, you are like diversified in here and we don't know really each other or like what their religious belief and all these things. It's advantageous if we learn what they want to eat, different culture, like somebody like, like the uh, you know, somebody cannot take like a blood you know transfusion whatever so it's, it's a very ... it will be informative and what kind of food and whatever practices FGI Medicine unit

I think the healthcare customs is very important, the religion is important, um, any ethnographic variables is important as well FGI Medicine unit

It could be worded nice and like, probably like I don't know, cultural beliefs on such and such, I don't know, Rather than dying ....your ending with doubt, and the death and dying part. Because this, you just said death and end of life is just like saying 'you're dying'. So probably, say culture beliefs on ...say you have a patient that you 
know is Catholic, and they know they're getting worse, ...Especially if you know they have specific, groups have specific religious, customs around death. Around dying... FGI Medicine unit

Finally, participants in this FGI suggested that the new tool should not be too complicated and should be restricted to basics.

\section{Yeah, I think with developing our own tool we should do it more basic... FGI Medicine unit}

One comment related more to the administration rather than content of the tool was provided by a FGI participant from the surgical unit:

I think we experience some of the patients were like 'what is this about', and I think um, if it was done right at the time of admission, it's not a foreign thing, cause it kind of goes along with the questions you're answering, but if you're doing it as a secondary thing FGI Surgery unit

The surgery unit nurses also commented that their current assessment tool was considered within the hospital to be very thorough, and that perhaps they could just embed some of the FamCHAT questions (particularly related to special considerations of culture and religion) into their current assessment. In opposition to one nurse's opinion in the Medicine unit, one in the surgery unit thought that education was perhaps a useful construct since it could help them craft their language in meaningful ways - towards a trained physician for instance.

\section{Discussion}

This study found that while the FamCHAT tool and the constructs contained within may have potential for helping to enhance cultural competency in nursing care by providing ethnoculturally specific information, it does require modification to meet the demands in the acute care working environment and the privacy concerns of patients. The optimal approach may be to embed the most useful constructs into the existing nursing care assessment framework, while considering that certain variables may be more relevant to some specialties (e.g. customs around birth in the women's unit and religious beliefs around blood transfusions in surgery).

The responses from the FamCHAT were not congruent with what was expected when considering this hospital's situation within a highly diverse neighbourhood where many immigrant communities reside. The variables of family size and language were not answered in accordance with the guidance notes on most instances. Moreover, questions that centered on food preferences/prohibitions, health care customs/practices related to death, birth, and illness and membership to formal and informal group membership were often left blank. Various factors could have contributed to these responses. There were time constraints to filling in a lengthy assessment tool, and the nurses felt short of time to explain or clarify questions with the patients. Despite attending the orientation sessions, it is possible that the participating nurses did not understand the process fully. It is unclear whether the nurses failed to read or understand the guide, or if their attempt to elicit this answer failed due to language barriers. An evaluation by the research team of the nurses' competency at eliciting the answers may have improved accuracy and response rates. The high rate of inaccuracy despite high levels of English comprehension of the patients tends to suggest that the terminology was not understood by the nurses or patients. Identifying which terms are most suitable for patients and nurses, and ways to define these in simple well-understood language would likely ensure more thorough completion of the form.

There was a clear indication of the value of having a good instrument or way to obtain specific information related to ethnocultural traditions and beliefs in an appropriate way from patients. While gaining knowledge of various ethnocultural groups can be important for health care professionals, clearly they cannot be expected to have full awareness of cultural practices of all types of religions and ethnic groups ${ }^{[20]}$. Gaining skills in "clinical cultural competency" (including the 
constructs of cultural sensitivity, awareness, and safety) is certainly critical and using tools to assist with understanding of and communication with patients important. Implementation of an assessment tool could be greatly enhanced by systemic efforts including the provision of a formal training program in cultural competency.

A dimension of cultural competency which this pilot study did not fully address is that at the organizational level. Organizational cultural competence occurs when the structures, processes and strategies operating within health care organizations help ensure the delivery of high quality care to achieve equitable outcomes to all clients regardless of their ethnocultural identity or socioeconomic status. Critical reflection on the inner workings of the organization is required and cultural competence at an organizational level involves the commitment of adequate resources to support appropriate responses at the service delivery level. Dreachslin, Weech-Maldonado, and Dansky ${ }^{[28]}$ and Ngo ${ }^{[29]}$ have developed models for describing and assessing organizational progress towards cultural competence. While this study was initiated by management having concerns of cultural competency, the implementation of an assessment tool is likely not to achieve substantial results without other considerations with respect to translation/interpretation services, referral systems, length of treatment and waiting times, and the predominance of the medical paradigm.

Access and utilization of health care services will be compromised if the patient and practitioner are not fluent in the official language or don't share another language ${ }^{[30,31]}$. Development and evaluation of standards of practice and models of delivery to improve language access to care have been recommended after review of the evidence on negative outcomes related to language barriers ${ }^{[32]}$. There is a general inadequacy of interpreting services resulting in reliance on family and friends to interpret, which can compromise the privacy and confidentiality of patients ${ }^{[32-34]}$. Moreover, effective communication requires an ability to interpret speech, tone, facial expressions, gestures, body language, and shared assumptions within a particular context and for a particular context ${ }^{[35,36]}$. Thus, nurses need to be able to adapt their strategies to respond to the cultural needs of their patients ${ }^{[35]}$.

One of the main concerns that were presented was the assumed "intrusion" into the private lives of patients, especially related to their beliefs and practices. Another serious concern that migrants may have, as reflected during the interviews, is the assumed threat to their stay in Canada that revelations as part of the assessment using the FamCHAT might create. these conceptualizations by some patients may considerably influence their responses to various questions. There was a great need to gain the trust of the patients with respect to anonymity when asking questions which can be perceived as too personal or risky for some people including refugees or asylum seekers.

When reviewing ideas expressed by the interviewees who are highly involved in patient assessment and care, it could be elicited that they support this tool, but with certain modifications. There were practical and constructive suggestions, which have emerged from administering the tool in their working environment. One key suggestion was perhaps that the constructs of most relevance to nursing practice (religion, family roles in health and death, cultural dietary practices) could be embedded in the existing nursing assessment framework to reduce time commitment (and some frustration related to duplicity) on the nurses' and patients' behalf. Suggestions to simplify the format were repeated and likely justified. Limiting some variables to assessments within specific specialties might be helpful to optimize relevance (e.g. customs around birth in the women's unit and religious beliefs around blood transfusions in surgery). Noteworthy though, is that it was apparent that the current nursing assessment form is completed largely by the lone patient without assistance with meaning or language. Language issues aside, enriching the assessment with additional questions related to religious and other cultural practices should be answered as accurately in the patient's perspective as possible using meaningful guidance if in the least to impart the anonymity and value of their information for personalized health care provision.

This study has limitations which are largely related to participant enrolment and compliance. Failure to gain better recruitment of nurse participants as well as have the participating nurse complete the forms with all patients was likely in part due to systemic issues. The regional health authority was at the time undergoing major restructuring to join within the newly formed provincial authority and associated staff shortages and reconfiguration meant that many pressures were 
placed on the nurses in addition to their usual duties. Additionally, critical clinical activities related to the N1H1 influenza were thought to also interfere with the compliance of the nurses at this time. These major issues were unavoidable and diminished our ability to obtain a larger, possibly more representative, patient sample. Another limitation evidenced by the lack of completeness of the forms, appears to be the comprehension of the nurses with respect to the construct definitions provided in the guidance notes and through the orientation sessions. The orientation sessions were provided to provide education and experiential learning of the constructs but it is possible that the attending nurses did not for unknown reasons ask for clarification.

\section{Conclusions}

This study highlights the importance of validating tools within specific settings prior to their implementation. Consideration is being given by the hospital management for integrating some of the constructs into their existing nursing assessment, with recognition that each unit might benefit from different approaches. Ethnocultural assessment belongs to a range of skills that nurses and other health care providers must have in order to provide culturally responsive care. Additionally, other systematic/organizational approaches to enhancing cultural competency also need to be considered.

\section{Conflicting interest}

The authors declare no conflicts of interest.

\section{Acknowledgement and funding}

The authors thank Dr Janice Davidson for allowing them to use and re-format the FamCHAT tool, Jennifer Pillay (Research Program Coordinator, Faculty of Nursing, University of Alberta, Canada; funded by Dr Gina Higginbottom's Canadian Institutes of Health Research Canada Research Chair in Ethnicity and Health) for her assistance with drafting and editing this manuscript, and Mohammad Salehyar (Research Assistant, Faculty of Nursing, University of Alberta, funded by the Royal Alexandra Hospital Nursing Research Foundation) for his contributions to the study.

\section{References}

[1] Beach CM, Green AG, Reitz JG (Eds). Canadian Immigration for the 21st Century. Montreal, ON: McGill/Queens University Press. 2003.

[2] Kymlicka W. Immigration, citizenship, multiculturalism: exploring the links. Polit Quart. 2003; 74s: 195-208. http://dx.doi.org/10.1111/j.1467-923X.2003.00590.x

[3] Citizenship and Immigration Canada. Facts and Figures: Immigration Overview Permanent and Temporary Residents 2008. Ottawa, ON: Author. 2009.

[4] Statistics Canada. Projections of the Diversity of the Canadian Population. Ottawa, ON: Demography Division, Statistics Canada. 2010.

[5] Statistics Canada. Canada’s Ethnocultural Mosaic, 2006 Census. Census year 2006. Ottawa, ON: Author. 2008.

[6] Health Disparities Task Group of the Federal/Provincial/Territorial Advisory Committee on Population Health and Health Security. Reducing Health Disparities- The Role of the Health Sector: Discussion Paper [Internet]. 2004. Available from: http://www.phac-aspc.gc.ca/ph-sp/disparities/pdf06/disparities_discussion_paper_e.pdf

[7] Public Health Agency of Canada. Chief Public Health Officer's Report on the State of Public Health in Canada 2008: Addressing Health Inequalities [Internet]. Ottawa: Author. 2008. Available from: http://www.phac-aspc.gc.ca/publicat/2008/cphorsphc-respcacsp/pdf/CPHO-Report-e.pdf

[8] Senate Subcommittee on Population Health. Population Health Policy: Issues and Options. Fourth Report of the Subcommittee on Population Health of the Standing Senate Committee on Social Affairs, Science and Technology [Internet]. 2008. Available from: http://www.parl.gc.ca/39/2/parlbus/commbus/senate/com-e/soci-e/rep-e/rep10apr08-e.pdf

[9] Schellenberg G, Maheux H. Immigrants' perspectives on their first four years in Canada Highlights from three waves of the longitudinal survey of immigrants to Canada (Catalogue No. 11-008). Ottawa: Statistics Canada, 2007. 
[10] Newbold KB. The short-term health of Canada’s new immigrant arrivals: evidence from LSIC. Ethn Health. 2009; 14 : 315-336. http://dx.doi.org/10.1080/13557850802609956

[11] Newbold KB. Self-rated health within the Canadian immigrant population: risk and the healthy immigrant effect. Soc Sci \& Med. 2005; 60: 1359-1370. http://dx.doi.org/10.1016/j.socscimed.2004.06.048

[12] Ng E, Wilkins R, Gendron F, Berthelot J-M. Health Today, Healthy Tomorrow? Findings from the National Population Health Survey. Dynamics of Immigrants' Health in Canada: Evidence from the National Population Health Survey (Catalogue no. 82-618-MWE2005002). Ottawa: Statistics Canada. 2005.

[13] Meddings F, Haith-Cooper M. Culture and communication in ethically appropriate care. Nurs Ethics. 2008 ; 15: 52-61. PMid:18096581 http://dx.doi.org/10.1177/0969733007083934

[14] Reitmanova S, Gustafson DL. "They can't understand it": maternity health and care needs of immigrant Muslim women in St John’s, Newfoundland. Matern Child Health J. 2008; 12: 101-111. PMid:17592762 http://dx.doi.org/10.1007/s10995-007-0213-4

[15] Betancourt JR. Cultural competence and medical education: many names, many perspectives, one goal. Acad Med. 2006; 81: 499-501. PMid:16728795 http://dx.doi.org/10.1097/01.ACM.0000225211.77088.cb

[16] Weissman JS, Bentacourt JR, Campbell EG. Resident physician’s -preparedness to provide cross-cultural care. JAMA. 2005; 294: 1058-1067. PMid:16145026 http://dx.doi.org/10.1001/jama.294.9.1058

[17] Keatings M, Smith OB. Ethical and Legal Issues in Canadian Nursing, 2nd Ed. Toronto: W.B. Saunders. 2000.

[18] Capell J, Dean E, Veenstra G. The relationship between cultural competence and ethnocentrism of health care professionals. J Transcult Nurs. 2008; 19: 121-125. PMid:18362206 http://dx.doi.org/10.1177/1043659607312970

[19] Giger JN, Davidhizar R, Purnell L, Harden JT, Phillips J, Strickland O. Understanding cultural language to enhance cultural competence. Nurs Outlook. 2007; 55: 212-214. http://dx.doi.org/10.1016/j.outlook.2007.05.004

[20] Betancourt JR. Cultural competency: providing quality care to diverse populations. The Consultant Pharmacist. $2006: 988-995$. PMid:17243850 http://dx.doi.org/10.4140/TCP.n.2006.988

[21] Collins E, Guruge S. Theoretical perspectives and conceptual frameworks. In Working with Immigrant Women: Issues and Strategies for Mental Health Professionals. S Guruge, E Collins, eds. Toronto, ON: Centre for Addiction and Mental Health. 2008, 19-38.

[22] Higginbottom GMA, Richter MS, Mogale RS, Ortiz Y, Young S, Mollell O. Identification of nursing assessment models/tools validated in clinical practice for use with diverse ethno-cultural groups: an integrative review of the literature. BMC Nursing. 2011; 10: 16. PMid:21812960 http://dx.doi.org/10.1186/1472-6955-10-16

[23] Leininger M. Transcultural nursing: the study and practice field. NSNA/Imprint. 1991; 38: 55-66.

[24] Weeks S, O’Connor P. The FAMTOOL family health assessment tool. Rehab Nurs. 1997; 22; 188-191. PMid:9275809

[25] Davidson JU, Regier T, Boos S. Assessing family cultural heritage in Kansas: research and development of the FamCHAT companion tool for family health assessment. Kansas Nurse. 2001; 76: 5-7.

[26] Statistics Canada. Ethnic Origin and Visible Minorities, 2006 Census Tables. Ottawa, ON: Author, 2008. Catalogue no. 97-562-XWE2006011.

[27] Roper J, Shapira J. Ethnography in Qualitative Research. London, UK: Sage Publications. 2000.

[28] Dreachslin JL, Weech-Maldonado R, Dansky KH. Racial and ethnic diversity and organizational behavior: a focused research agenda for health services management. Soc Sci \& Med. 2004; 59: 961-971. http://dx.doi.org/10.1016/j.socscimed.2003.12.007

[29] Ngo H. Cultural Competency: A Self-Assessment Guide for Human Service Organizations. Calgary, AB: Cultural Diversity Institute. 2000.

[30] Pharoah C. Primary Health Care for Elderly People from Black and Minority Ethnic Communities. London: HMSO. 1995.

[31] Ahmad WIU. Ethnicity, Disability and Chronic Illness. Buckingham: Open University Press. 2000.

[32] Bowen S. Language Barriers in Access to Health Care. Ottawa: Health Canada. 2001.

[33] Gerrish K. The nature and effect of communication difficulties arising from interactions between district nurses and South Asian patients and their carers. J Adv Nurs. 2001; 33: 566-574. PMid:11298192 http://dx.doi.org/10.1046/j.1365-2648.2001.01674.x

[34] Bhakta P, Katbamna S, Parker G. South Asian carers' experiences of primary health care teams. In Ethnicity, Disability and Chronic Illness. WIU Ahmad, ed. Buckingham: Open University Press. 2000; 123-138.

[35] Jirwe M, Gerrish K, Emami A. Student nurses’ experiences of communication in cross-cultural care encounters. Scand J Caring Sci. 2010; 24: 436-444. PMid:20233352 http://dx.doi.org/10.1111/j.1471-6712.2009.00733.x

[36] Bradby H. Communication, interpretation and translation. In Ethnicity and Nursing Practice. Culley, S Dyson, eds. Palgrave: Basingstoke. 2001; 129-148.

[37] Leininger M. Overview of the theory of culture care with the ethnonursing research method. J Trans Nurs. $1997 ; 8$ : 32-52. http://dx.doi.org/10.1177/104365969700800205 
[38] Kim-Godwin YS, Clarke PN, Barton L. A model for the delivery of culturally competent community care. J Adv Nurs. 2001; 35: 918-925. PMid:11555040 http://dx.doi.org/10.1046/j.1365-2648.2001.01929.x

[39] Narayanasamy A. The ACCESS model: a transcultural nursing practice framework. Brit J Nurs. 2002; 11: 643-650. PMid:12048479

[40] Campinha-Bacote J. A model and instrument for addressing cultural competence in health care. J Nurs Educ. 1999 ; $38: 204-207$.

[41] Purnell L, Paulanka B. Transcultural Health Care: A Culturally Competent Approach. Philadelphia: FA Davis. 1998.

[42] Papadopoulos I, Tilki M, Taylor G. Transcultural Care. A Guide for Health Care Professionals. Dinton, Wilts: Quay Publications, 1998.

[43] Giger JN, Davidhizar RE. Transcultural nursing assessment: a method of advanced nursing practice. Int Nurs Rev 1990; 37: 199-201. PMid:2312240 